\title{
High-Resolution X-ray Imaging Based on Curved Bragg Mirrors: First Results
}

\author{
Uwe Bergmann, Marijana Ivanovic, Member, IEEE, Pieter Glatzel, and Stephen P. Cramer
}

\begin{abstract}
Small animal cancer imaging has drawn increased attention over the last few years due to greater availability of genetically modified mice, permitting the study of human diseases in animal models. Submillimeter resolution would be of great value to provide the fine detail needed in the imaging of small tumors traced by radio-labeled agents. Despite extensive research and improvements in instrumentation and imaging reconstruction, until now, there has been no efficient technology for this task. The limitations of scintillation cameras with pinhole collimators, currently the highest resolution devices, are fundamental in nature. They include image blurring through edge effects, scattering at the pinhole, and inelastic Compton scattering. Furthermore, such devices often yield low efficiency. In this paper, a new approach to high-resolution imaging of radio-labeled agents is introduced and first results are shown. The technique is based on curved perfect-crystal X-ray mirrors applied in a one-to-one focusing geometry. Such Bragg diffraction optics yields high reflectivity and excellent energy resolution and it has been applied in X-ray spectroscopy for many years. Today large perfect-crystal mirrors are commercially available and efficient devices covering a substantial solid angle can be envisioned. The potential advantage over conventional pinhole cameras is twofold. First, focusing diffraction optics provides a "virtual" pinhole, which can effectively be inside the object under investigation and does not suffer from edge effects. Second, Bragg optics has an energy resolution of a few $\mathrm{eV}$ and discriminates against Compton scattering. The fundamentals of Bragg optics are dicussed and first results using ${ }^{55} \mathrm{Fe}$ and ${ }^{95 m} \mathrm{Tc}$ phantoms are presented. Our data show spatial resolutions of less than 1 and $2 \mathrm{~mm}$, respectively. Current limitations of this new technique and possible future designs are discussed.
\end{abstract}

Index Terms-Biomedical imaging, Bragg reflection, small animal cancer imaging, X-rays, X-ray detectors, X-ray scattering.

\section{INTRODUCTION}

$\mathbf{H}$ IGHER spatial resolution and sensitivity for small animal imaging is continuously sought to improve the spatial detail and image quality that can be obtained in radionuclide imaging. Radionuclide imaging has profited from greater tissue specificity and highly selective uptake of many of the new labeled monoclonal antibodies, receptor specific molecules, peptides, and other new radiopharmaceuticals [1]-[4]. These

Manuscript received January 3, 2002; revised August 15, 2002. This work was supported in part by the Department of Health and Human Services, NIH National Cancer Institute1 R21, under Grant 87499-01.

U. Bergmann and S. P. Cramer are with the Lawrence Berkley National Laboratory, Berkley, CA 94720, USA, and also with the Department of Applied Science, University of California, Davis, Davis, CA 95616 USA (e-mail: ubergmann@lbl.gov).

M. Ivanovic is with the Department of Radiology, University of North Carolina, Chapel Hill, NC 27514 USA.

P. Glatzel is with the Department of Applied Science, University of California

Davis, Davis, CA 95616 USA.

Digital Object Identifier 10.1109/TNS.2002.807884 advances also place new demands on imaging with high spatial resolution in order to characterize regional localization and turnover properties.

Planar imaging of the activity distribution of radiopharmaceuticals in small animals has been conventionally performed using scintillation cameras with high-resolution, parallel hole collimators or pinhole collimators. Although good spatial resolution can be achieved with these techniques, only two-dimensional images of three-dimensional activity distributions are obtained and spatial detail and contrast are limited due to crosstalk from activity in overlying or underlying tissues. Spatial resolution of the order of 6-10 mm is obtained with high-resolution parallel hole collimators and $2-5 \mathrm{~mm}$ with pinhole collimators. SPECT imaging with conventional parallel hole, fan, or cone beam collimators can provide tomograms of the activity distribution in three dimensions, however, the spatial resolution achieved with these collimators is usually not adequate to provide the fine detail needed for many tissues and organs in small laboratory animals. Spatial resolution of 1-3 mm is often sought for these studies. As a result, considerable effort has been given to identify and characterize new detectors and to modify more conventional detectors to achieve greater spatial resolution and adequate sensitivity [5]-[10].

In this work, we present first studies of a new approach to high-resolution imaging of radio-labeled agents. The technique is based on focusing X-ray optics using perfect crystal Bragg mirrors. In the visible range imaging devices such as mirrors, lenses, and wave-guides rely on the large refraction index of glass or other materials. Due to the short wavelength of hard $\mathrm{X}$-rays and $\gamma$-rays condensed matter has, at these high frequencies, generally an index of refraction somewhat smaller than, but very close to one. As a consequence the critical angle for total reflection is very small (of order mrad). Considering absorption and stringent requirements on surface quality, focusing X-ray optics based on many total reflections are not very efficient in the hard X-ray regime. (Although there is an increased use of capillary X-ray optics based on total reflection in the soft and medium X-ray range).

A different way to reflect X-rays at much larger angles makes use of diffraction from crystalline material. This so-called Bragg optics is commonly used in X-ray spectroscopy. Large perfect single crystals such as $\mathrm{Si}$ or $\mathrm{Ge}$ are grown routinely nowadays, and curved X-ray Bragg mirrors have become larger as well [11]. Due to the nature of Bragg reflections a curved Bragg mirror can act like a monochromator and lens at the same time. Thus, different from a $\gamma$-camera based on a real pinhole, a focusing Bragg mirror can provide a "virtual" pinhole. In the following section, we describe the principle of Bragg optics and in 


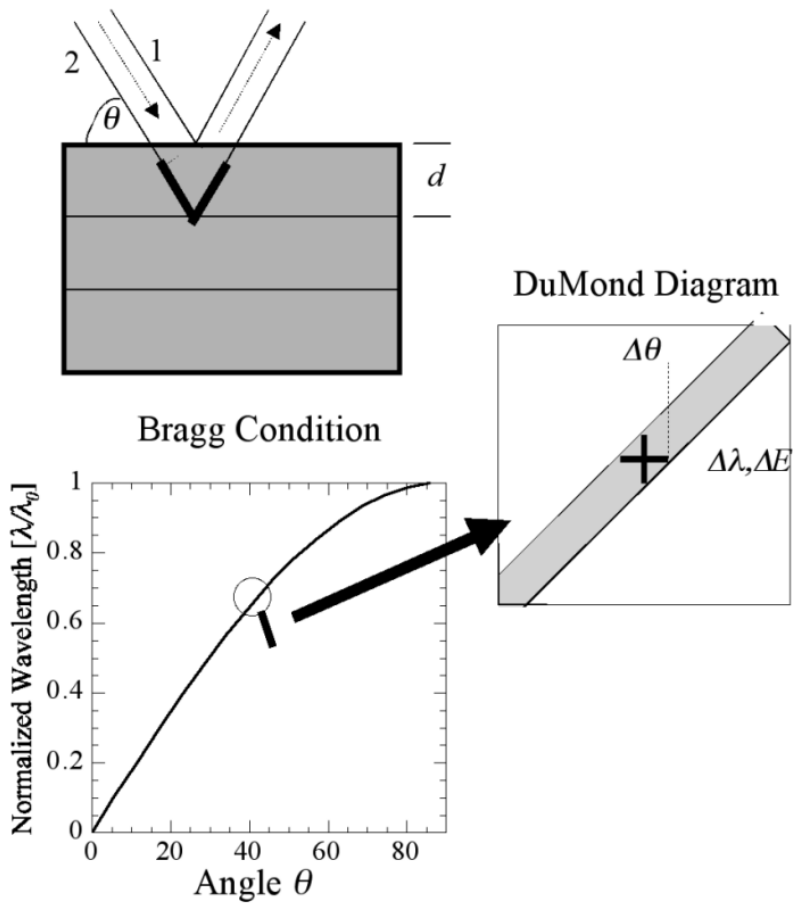

Fig. 1. Basics of Bragg reflection. Top left: Geometrical view of Bragg condition. The thick line indicating the path difference of two rays, 1 and 2, has to be equal to their wavelength, $\lambda$. The distance of adjacent crystal planes is indicated by $d$. Bottom left: Plot of Bragg's law. The wavelength is normalized to $\lambda_{0} \equiv 2 d$. Right: DuMond diagram to indicate the relation between Darwin width, $\Delta \theta$, and energy resolution $\Delta E$. The Darwin width is calculated by dynamical theory.

Section III, we show first results to test the imaging resolution of such a system. We conclude by discussing current limitations and possible future designs.

\section{THEORY AND PRINCIPLE}

\section{A. Fundamentals of Bragg Optics, Flat Mirror}

The X-ray mirror system described in this study is based on curved Bragg crystals. Nevertheless, the fundamentals of Bragg reflections are best explained with a flat Bragg mirror. The results can then be applied to curved mirror optics.

A Bragg reflection is a diffraction phenomenon where under a condition known as Bragg's law, X-rays impinging on a crystal are reflected. This condition is achieved when the path difference of X-rays reflected by two neighboring diffraction planes equals their wavelength (Fig. 1 top left). It is given by the equation

$$
2 d \sin \theta=\lambda
$$

with the Bragg angle $\theta$, the wavelength $\lambda$ and the $d$-spacing $d$ indicating the distance of neighboring diffraction planes. For a cubic crystal $d=a /\left(h^{2}+k^{2}+l^{2}\right)^{1 / 2}$, where $a$ is the length of the unit cell and $h, k, l$ are the Miller indices. ${ }^{1}$ Fig. 1 bottom left shows the sinusoidal dependence between wavelength and angle

${ }^{1}$ Strictly spoken, the Bragg equation has an integer factor $n$ in front of the wavelength to indicate that the path difference has to be equal to a multiple of the wavelength. For radionuclide imaging integer multiple wavelengths basically never occur, and we therefore do not need to address this phenomenon. according to the Bragg (1), with the wavelength normalized to $\lambda_{0} \equiv 2 d$.

It is this relation between wavelength (or energy) and angle, which sets apart a Bragg mirror from a conventional imaging device. At a given wavelength a Bragg mirror only reflects at an angle $\theta$ determined by (1). More precisely, there is a small angular range $\Delta \theta$ in which rays with wavelength $\lambda$ are reflected. This angular range $\Delta \theta$ is called Darwin width and is calculated by solving the Maxwell equations in a periodic dielectric medium. Within the angular range of the Darwin width, an incident X-ray wave cannot travel inside the crystal and is therefore reflected. This theoretical treatment has been successfully applied since many decades and is generally known as dynamical theory, see e.g., [12]-[15]. The Darwin width depends on the wavelength, Bragg angle and reflection order, as well as the crystal material. In general, high order reflections (large Miller indexes) and mirrors made out of low $Z$ materials have narrow Darwin width. There are numerous programs available for such calculations, e.g., XOP (URL: http://www.esrf.fr/computing/scientific/xop/). An instructive way to visualize the relation between Darwin width and energy resolution of a Bragg mirror is by a so-called DuMond diagram shown in Fig. 1 right [16]. The symbolized magnifying glass indicates that in a DuMond diagram only a tiny fraction of the whole Bragg curve is shown, because typical Darwin widths are very small of order arcsec.

The shaded area in the DuMond diagram represents the phase space in which X-rays are reflected from a Bragg mirror. The Darwin width, $\Delta \theta$, is indicated by the horizontal line and corresponding to that is the energy width, $\Delta E$, (or wavelength spread $\Delta \lambda$ ) indicated by the vertical line. To show how to apply a DuMond diagram to estimate the reflection properties of a Bragg crystal we will consider two cases. First, however, we give another important general equation between energy width $\Delta E$ and angular width $\Delta \theta$ of a Bragg mirror. It is the derivative of (1), resulting in

$$
\left|\frac{\Delta E}{E}\right|=\left|\frac{\Delta \lambda}{\lambda}\right|=\Delta \theta \cot \theta
$$

In the first case, we consider a perfectly monochromatic $(\Delta \lambda=$ $\Delta E=0)$ point-like $\gamma$-ray source. In DuMond space the source is represented by a horizontal line, because the angular spread is very large. In that case, the X-ray mirror only reflects a small angular range, given by the overlap of this line with the shaded area, i.e., the Darwin width $\Delta \theta$. Consequently, the spatial resolution of a Bragg mirror imaging a monochromatic point source is determined by the Darwin width and its distance from source and detector.

Second, we consider a point source with an energy width much larger than $\Delta E$, e.g., a $K \alpha \mathrm{X}$-ray line. In the DuMond diagram this situation corresponds to a horizontal band with a vertical spread given by that energy width. The phase space reflected by the mirror is again represented by the overlap with the shaded area, and the angular width of this overlap area is now much larger than the Darwin width $\Delta \theta$. It can be obtained graphically with a DuMond diagram, or equivalently calculated by (2) when replacing $\Delta E$ with the energy width of the X-ray line. Consequently the spatial resolution of imaging a point source of 


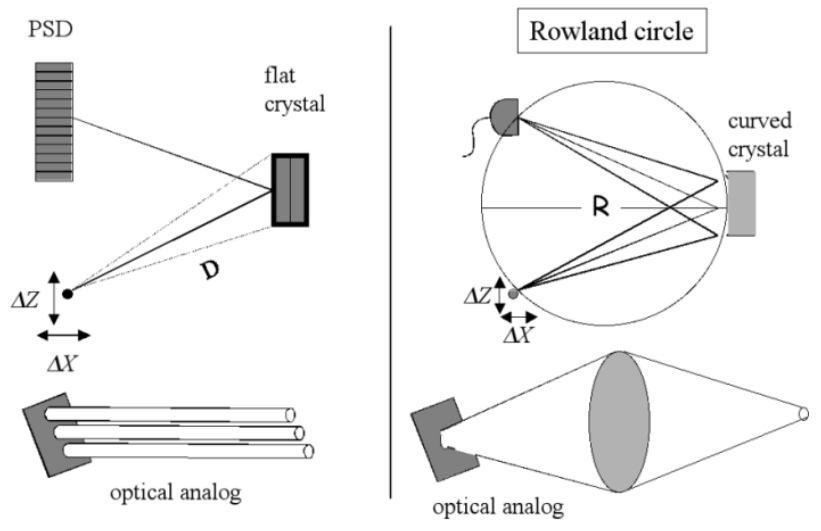

Fig. 2. Flat versus curved mirror. Left: From a monochromatic source, a flat Bragg mirror only reflects the center ray fulfilling the Bragg condition. The mirror acts essentially like a set of parallel hole collimators as shown on the bottom. Right: Ideally all rays from a point source impinge on a curved mirror in Rowland geometry at the same angle. They are reflected when the Bragg condition is fulfilled. The mirror essentially acts as a one-to-one imaging lens as shown on the bottom.

a broad X-ray line is no longer determined by the Darwin width of the mirror but rather by the angular width obtained from (2).

To calculate the spatial resolution of a flat Bragg mirror system consider a two-dimensional source extended in the $X$ and $Z$ directions (Fig. 2) and imaged with a position sensitive detector (PSD) with very small pixel pitch. The spatial resolution of such an imaging system $\Delta X, \Delta Z$ is determined through

$$
\Delta Z=\frac{2 D \Delta \theta}{\sin (\theta)}, \Delta X=\frac{2 D \Delta \theta}{\cos (\theta)}
$$

where $D$ is the distance from source to mirror and $\Delta \theta$ is determined according to the discussion above. (The resolution $\Delta Y$ perpendicular to the drawing plane is determined by second order effects and is much poorer.) Such a system acts similar to a parallel hole collimator camera. Assuming a very small pixel size for the PSD ( $<100 \mu \mathrm{m}$ is routinely available), the spatial resolution along $Z$ and $X$ can be quite good. For instance, at $\theta=45^{\circ}$, the $27 \mathrm{keV}$ Te $K \alpha_{1}$ line following ${ }^{125} \mathrm{I}$ decay has an estimated energy width of $\Delta E=50 \mathrm{eV}$ resulting in $\Delta E / E=\Delta \theta=1.9 * 10^{-3}$ and at a source-mirror distance of $D=100 \mathrm{~mm}$, the resulting spatial resolution would be $\Delta Z=\Delta X=0.54 \mathrm{~mm}$. For Si or Ge crystals, the contributions from the Darwin width would be negligible in this case.

Nevertheless, a flat X-ray mirror is impractical as an imaging device. First and most importantly, the accepted solid angle is extremely small (typically of order some arcsec) making the device very inefficient. Second, X-ray lines such as $K \alpha$ fluorescence are usually split into sublevels (in the case of Te the $K \alpha_{1}-K \alpha_{2}$ splitting is $\sim 270 \mathrm{eV}$ ) which would create a double image. Finally, Compton scattering would create blurring of the image, because it increases $\Delta E$ and, hence, $\Delta \theta$.

\section{B. Fundamentals of Bragg Optics, Curved Mirror}

These limitations can be overcome by using either a large array of small flat Bragg mirrors [17] or, as discussed in the fol- lowing, a system based on curved mirrors. In the Rowland circle approach (Fig. 2 right), a mirror is curved to a shape for which each ray from a point source ideally impinges on the mirror at the same angle and is refocused to one and the same image point. This can be achieved with the so-called Rowland condition, when source, mirror, and detector lie on a circle whose diameter is identical to the radius of curvature, $R$, of the diffraction planes of a curved mirror. When detector and mirror are aligned to a Bragg angle $\theta$ corresponding to the source energy, the Rowland-circle mirror acts like a one-to-one focusing lens.

The precise Rowland condition can be obtained with a so-called Johansson type crystal [18]. Here the crystal surface has a radius of curvature of $R / 2$ ('touching' the Rowland circle), and the diffraction planes are curved to a radius $R$. However, Johansson devices are extremely difficult to manufacture. Therefore, Johann type [19] crystals commonly are used where the surface is parallel to the diffraction planes curved at radius $R$. In this case, the surface of the mirror touches the Rowland circle only in the center and consequently some aberrations contributing to both spatial and energy resolution occur.

If these aberrations are small, the spatial resolution of Rowland optics can be estimated as follows:

- calculate the angular range $\Delta \theta$ over which the Bragg mirror reflects. For a monochromatic source this is just the Darwin width (extreme case 1); for an X-ray line, this is given by (2) using for $\Delta E$ the width of the $\mathrm{X}$-ray line (extreme case 2).

- calculate the spatial resolution $\Delta Z, \Delta X$ corresponding to $\Delta \theta$ with (see Fig. 2):

$$
\Delta Z=R \Delta \theta, \Delta X=R \Delta \theta \tan (\theta)
$$

The resolution can be improved further by using an aperture slit in front of the detector. Then, ideally, the aperture defines the resolution. Such an aperture is necessary when either the $\mathrm{X}$-ray line is too broad or split (e.g., $K \alpha_{1} / K \alpha_{2}$ ), or Compton scattering is significant. These "wrong" energies are reflected at "wrong" Bragg angles thus impinging on the detector outside the focal point chosen for the source energy. The aperture then discriminates against these rays improving the spatial resolution with a corresponding loss of geometric efficiency.

In principle two setups with Rowland optics can be used. First, the "scanning mode," where source and detector are in the focal point (as shown in Fig. 2). Second, the "camera mode," where the source is imaged onto a PSD and both source and PSD are outside the focal plane. The "scanning mode" has the advantage that with an aperture slit it discriminate against "wrong energies" and therefore provides better spatial resolution. The "camera mode" has the advantage that an image can be recorded at once, which might lead to a higher efficiency. As compared to a conventional $\gamma$ camera, the difference is that the focus of the Rowland optics is based on a "virtual" slit, avoiding problems related to real slits. The advantage over the flat mirror design is a much larger angular acceptance (degrees versus arcsec), and the complete rejection of Compton scattering and split sublevels. In summary, the optimal curved Bragg mirror in Rowland geometry would have the following features. 




Fig. 3. Multicrystal imaging array. Spherically curved Ge or Si crystal with $9 \mathrm{~cm}$ diameter, and $86 \mathrm{~cm}$ radius of curvature. Ideally, only X-rays from a source matching the focal point are detected. The instrument shown here is not able to image the third dimension with good resolution, since angles perpendicular to the reflection plane only change the Bragg condition in second order.

- Bragg reflection with a Darwin-width, $\Delta \theta$, approximately matching the energy width of the X-ray line used for imaging through (2) and the desired spatial resolution through (4). This provides maximum single mirror efficiency.

- Bragg angle of $\theta \approx 45^{\circ}$ to match spatial resolution $\Delta X$ and $\Delta Z$.

- Johansson crystals to minimize image aberrations.

- Multiple mirrors to achieve largest device efficiency

\section{EXPERIMENTAL RESULTS AND DISCUSSION}

The instrument used in the studies shown here was originally designed as a spectrometer to achieve best energy resolution and was optimized to operate at higher order Bragg reflections with Bragg angles close to $\theta=90^{\circ}$. It is based on up to eight spherically curved Johann type [19] Si or Ge crystals aligned on intersecting Rowland circles (Fig. 3). For X-ray lines, the choice of backscattering Bragg angles is clearly a compromise to the spatial resolution. Furthermore, Si and Ge Bragg reflections have very narrow Darwin widths, corresponding to energy resolutions that are much better than that of the X-ray line (extreme case two). This limits the mirror efficiency. Nevertheless, the device is well suited to test the concept of Bragg mirror imaging systems.

A detailed description of the instrument is given in [20] and only some details are noted here. The alignment is done with computer controlled stepper motors with an angular precision better than $35 \mu \mathrm{rad}$ and an absolute spatial positioning of $<0.1 \mathrm{~mm}$. The detector is a liquid nitrogen cooled Ge photon counting detector (Canberra, Meriden, CT) with subsequent pre-amplifier, amplifier, single channel analyzer, and read out computer. The aperture slit in front of the detector is made out of lead with horizontal aperture of $15 \mathrm{~mm}$ and varying vertical aperture.

Phantoms based on two different sources were measured. First, a ${ }^{55} \mathrm{Fe}$ K-capture source emitting predominantly $\mathrm{Mn}$ $K \alpha \mathrm{X}$-rays at $5.9 \mathrm{keV}$. The source was placed behind an opening slit of vertical size $\Delta z=0.4 \mathrm{~mm}$ and horizontal

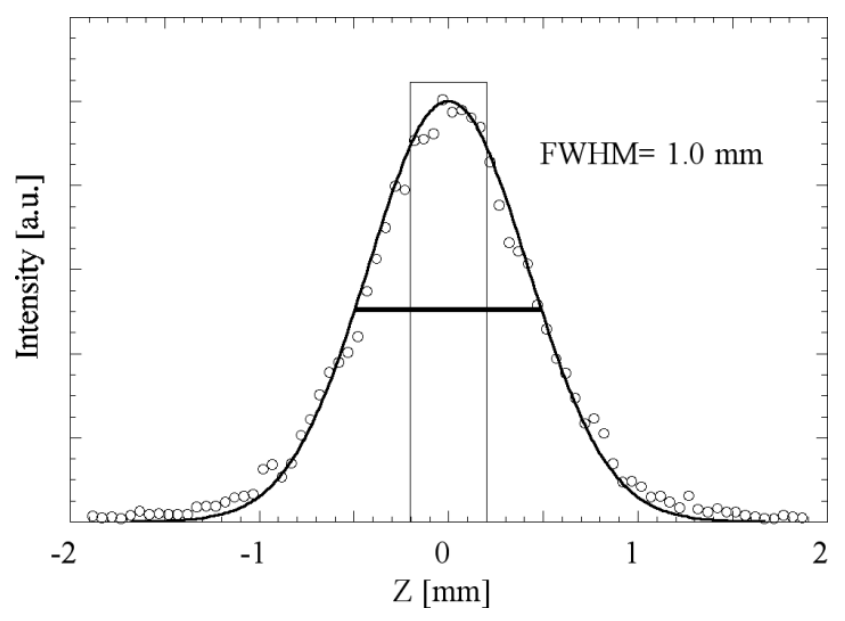

Fig. 4. Imaging of a $0.4 \mathrm{~mm}^{55} \mathrm{Fe}$ source at $5.9 \mathrm{keV} \mathrm{Mn} K \alpha_{1}$ radiation. Data are fitted by a Gaussian with $1 \mathrm{~mm}$ FWHM (solid line). The $0.4 \mathrm{~mm}$ slit function is drawn for comparison.

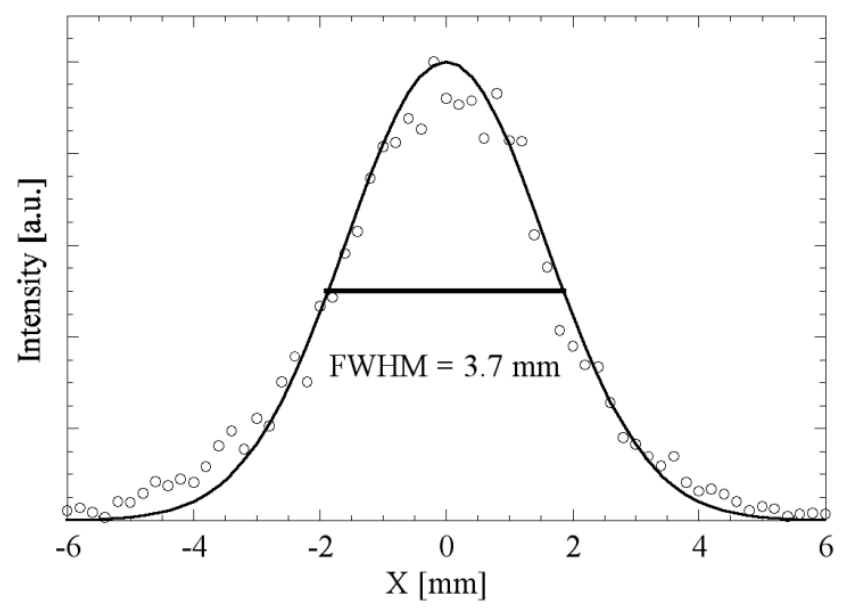

Fig. 5. $X$-scan of ${ }^{55} \mathrm{Fe}$ phantom imaging the $\mathrm{Mn} K \alpha_{1}$ radiation. Data are fitted by a Gaussian with $3.7 \mathrm{~mm}$ FWHM (solid line).

size $\Delta y=10 \mathrm{~mm}$. The depth of the source is estimated to be $\Delta x<15 \mu \mathrm{m}$ (absorption length in $\mathrm{Fe}$ ) and, therefore, negligible on the imaging scale studied here. An array of four Ge $(3,3,3)$ crystals (center four crystals of Fig. 3 ) is operated at $\theta=74.85^{\circ}$ ( $K \alpha_{1}$ energy), and a $0.3 \mathrm{~mm}$ vertical aperture slit is placed in front of the detector. The solid angle captured in this setup is $0.25 \%$ of $4 \pi$ sr. Phantom scans were performed along $Z$ and $X$ using a computer controlled motorized sample stage. Fig. 4 shows the scan along $Z$. The resulting image can be fitted with a Gaussian of $1 \mathrm{~mm}$ FWHM corresponding to an instrumental $Z$-resolution of $0.9 \mathrm{~mm}$ FWHM (from deconvolution of a $4 \mathrm{~mm}$ rectangular function with a $1 \mathrm{~mm}$ Gaussian).

In Fig. 5 the same phantom is scanned along the $X$-direction. The obtained resolution is $3.7 \mathrm{~mm}$. From (4) it follows that for a single mirror device $\Delta X / \Delta Z=\tan (\theta)$, hence at $\theta=74.85^{\circ}$, $\Delta X / \Delta Z=3.7$, which is very close to our measured ratio.

We now compare the measured resolution with the calculated value (no aperture slit). Following (2) the angular range in which the mirror reflects the $\Delta E=4 \mathrm{eV}$ broad Mn $K \alpha_{1}$ line (extreme case two) is $\Delta \theta=2.5 \mathrm{mrad}$. Using $R=860 \mathrm{~mm}$ in 


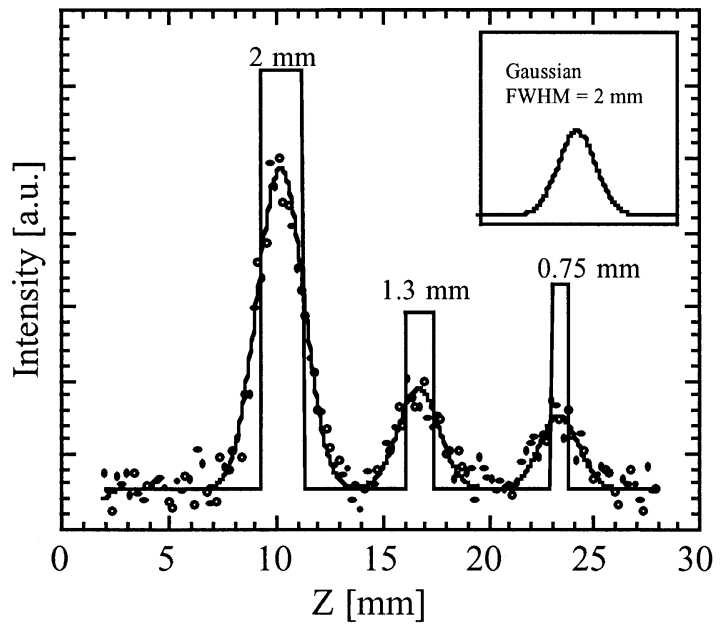

Fig. 6. Imaging of a ${ }^{95 m} \mathrm{Tc}$ phantom with variable size sources using $19.6 \mathrm{keV}$ Mo $K \beta$ radiation. The slit function is drawn for comparison.

(4) the vertical spatial resolution becomes $\Delta Z=2.1 \mathrm{~mm}$. This shows that, as expected, the aperture slit enhances the resolution. In fact, for an ideal mirror we would expect a $Z$ resolution solely determined by the aperture slit $(0.3 \mathrm{~mm})$. The measured value of $0.9 \mathrm{~mm}$ is due to Johann mirror aberrations and possibly some contributions from the $Y$ dimension of the phantom. Nevertheless, it shows that the Bragg mirror is well suited for high-resolution imaging.

To estimate the efficiency of this system we have to compare the calculated Darwin width, $\Delta \theta=78 \mu \mathrm{rad}$, with the angular range of $2.5 \mathrm{mrad}$ stemming from the width of $K \alpha_{1}$. This large difference indicates that with mirrors of matching Darwin width, the efficiency of could be improved by a factor of 30 without loss of imaging resolution, if a matching X-ray mirror could be found. We are currently studying suitable crystal with larger atomic numbers, which have broader Darwin widths.

In a second experiment, using a more relevant source we imaged a phantom at the $19.6 \mathrm{keV}$ Mo $K \beta$ fluorescence, following the decay of ${ }^{95 m} \mathrm{Tc}$. Tc is a commonly used emitter and at $\sim 20 \mathrm{keV}$ the absorption length in water is $\sim 15 \mathrm{~mm}$, sufficient for small animal imaging. An array of $8 \mathrm{Si}(12,12,0)$ crystals was operated at $\theta=81^{\circ}$ capturing $0.5 \%$ of $4 \pi$ sr. The phantom consisted of an array of three slits cut into a lead sheet (see Fig. 3). The vertical dimensions were openings of 2, 1.3, and $0.75 \mathrm{~mm}$ spaced by 4.65 and $5.5 \mathrm{~mm}$, respectively. The horizontal opening was $13 \mathrm{~mm}$ in all three slits. The vertical aperture in front of the detector was $3 \mathrm{~mm}$. Three vials containing a solution of ${ }^{95 m} \mathrm{Tc}$ were placed behind each slit, respectively. The total activity contributing X-rays that exit the phantom slits was not measured, but is estimated at less than $0.1 \mathrm{mCi}$.

Fig. 6 shows the background subtracted average of multiple $Z$-scans of the phantom, with a total counting time of $3.3 \mathrm{~h}$ per data point. A fit to the data yields a Gaussian resolution function of $2 \mathrm{~mm}$ FWHM. The calculated value without aperture slit for the $\Delta E \sim 9 \mathrm{eV}$ broad Mo $K \beta_{1}$ line is $\Delta Z=2.9 \mathrm{mrad} *$ $860 \mathrm{~mm}=2.5 \mathrm{~mm}$. This shows that the aperture slit does not affect the measured resolution, it does, however, cut off the $K \beta_{3}$ line (which would cause a double image) and possible Compton scattering.

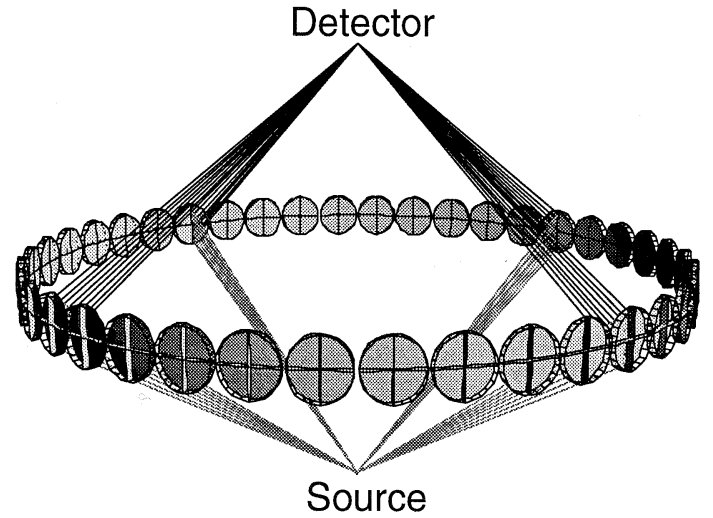

Fig. 7. Full circle curved Bragg mirror array. At the appropriate Bragg angle the optics can provide good spatial resolution in all three dimensions.

The fact that our measured resolution is even somewhat below the calculated number can be explained by the simplifications in (2). It considers neither the exact shape of the $K \beta_{1}$ line nor that of the Darwin curve.

In principle we could improve the resolution by narrowing the detector aperture and a mirror optimized for Mo $K \alpha$ would enhance the signal by a factor eight (ratio of $K \alpha$ versus $K \beta$ fluorescence yield). However, the by far largest improvement could come from a Bragg mirror having its Darwin width matched to the spectral width of the X-ray source. The $\mathrm{Si}(12,12,0)$ Darwin width of $\Delta \theta=1.2 \mu \mathrm{rad}$ is more than three orders of magnitude narrower than needed (the angular width corresponding to the Mo $K \beta_{1}$ line is $2.9 \mathrm{mrad}$ ), and this factor is lost in imaging efficiency.

\section{CONCLUSION AND OUTLOOK}

A new approach to high-resolution medical imaging based on curved Bragg mirror optics has been discussed and first results were shown. At this early stage of research and development, with a suboptimal system, imaging at below $1 \mathrm{~mm}$ resolution has been demonstrated. From these results it is apparent that spatial resolution will not be the limiting factor of this technique. In fact, much better resolution should be obtainable just by choosing a system operating at smaller Bragg angles. The more difficult challenge lies in the improvement of efficiency. It is clear from the current study that $\mathrm{Si}$ and Ge mirrors have Darwin widths that are far too narrow to match most of the X-ray lines and the required spatial resolution of order $1 \mathrm{~mm}$. There are some narrow $\gamma$-lines such as the $35 \mathrm{keV}$ transition of ${ }^{125} \mathrm{I}$ or the $23.8 \mathrm{keV}$ transition of ${ }^{119} \mathrm{Sb}$, which might be good candidates for these materials. But even in this case, the efficiency would be far from optimal because then, the limiting factor to the resolution would solely come from aberrations. Therefore Bragg mirrors made out of crystals with much larger Darwin widths are needed. Smither and Roa have shown that $\mathrm{Cu}$ might be a good candidate for imaging at higher energy X-rays (100-250 keV) [19], but at this point it is not clear to us what the ultimate material would be. One might think of mosaic crystals or even multilayers.

In addition to finding the appropriate mirror material, the acceptance angle of the imaging system has to be maximized. Fig. 7 shows a possible design, employing a full circle of curved 
Bragg mirrors. Besides a larger solid angle, this design has the advantage of providing good resolution in all three dimensions. From the cylindrical symmetry it is obvious, that the resolutions along $X$ and $Y$ are identical, and at a Bragg angle of $45^{\circ}$ this would match the resolution along $Z$. The efficiency can be further improved by adding more concentric circles of mirrors. Such a device could eventually be used to efficiently image small animal tumors with extremely high spatial resolution, thus complementing more conventional systems.

\section{REFERENCES}

[1] D. A. Weber, K. F. Eckerman, L. T. Dillman, and J. C. Ryman, "MIRD Radionuclide data and decay schemes," Soc. Nucl. Med., p. 447, 1989.

[2] A. N. Serafini, "From monoclonal antibodies to peptides and molecular recognition units: An overview," J. Nucl. Med., vol. 34, pp. 533-536, 1993.

[3] D. A. Weber and A. I. Kassis, Eds., "Dosimetry of radiolabeled antibodies," in Med. Phys., 1993, pt. II, vol. 20.

[4] S. Srivastava, "Is there life after technetium: What is the potential for developing new broad-based radionuclides?," Sem. Nucl. Med., vol. 26, pp. 119-131, 1996

[5] D. A. Weber et al., "Pinhole SPECT: An approach to in vivo high resolution SPECT imaging in small laboratory animals," J. Nucl. Med., vol 35, pp. 342-348, 1994.

[6] R. J. Jaszczak, J. Li, H. Wang, M. R. Zalutsky, and R. E. Coleman, "Pinhole collimation for ultra-high-resolution, small-field-of-view SPECT," Phys. Med. Biol., vol. 39, pp. 425-437, 1994.

[7] S.-E. Strand, M. Ivanovic, K. Erlandsson, D. Franceschi, T. Button, K. Sjögren, and D. A. Weber, "Tumor imaging with pinhole SPECT," Cancer Supplement, vol. 73, pp. 981-984, 1994.
[8] G. S. Kastis, M. C. Wu, S. J. Balzer, D. W. Wilson, L. R. Furenlid, G. Stevenson, H. B. Barber, H. H. Barrett, J. M. Woolfenden, P. Kelly, and M. Appleby, "Tomographic small-animal imaging using a high-resolution semiconductor camera," in Proc. IEEE MIC'01, vol. 21, San Diego, CA, 2001, pp. 31-35.

[9] S. R. Meikle, R. R. Fulton, S. Eberl, M. Dahlbom, K. P. Wong, and M. J. Fulham, "An investigation of coded aperture imaging for small animal SPECT," in Proc. IEEE MIC'01, vol. 21, San Diego, CA, 2001, pp. 81-85.

[10] M. C. Wu, G. A. Kastis, S. J. Balzer, D. W. Wilson, H. B. Barber, H. H. Barrett, M. W. Dae, and B. H. Hasegawa, "High-resolution SPECT with a CdZnTe detector array and a scintillation camera," in Proc. IEEE MIC'01, vol. 21, San Diego, CA, 2001, pp. 76-80.

[11] V. Stojanoff, K. Hamalainen, D. P. Siddons, J. B. Hastings, L. E. Berman, S. P. Cramer, and G. Smith, "A high-resolution fluorescence spectrometer for near-edge absorption studies," Rev. Sci. Instrum., vol. 63, pp. $1125-1127,1992$

[12] W. H. Zachariasen, Theory of X-Ray Diffraction in Crystals. New York: Wiley, 1945.

[13] R. W. James, Optical Principles of the Diffraction of X-Rays. London, U.K.: Bell, 1950

[14] _ "The dynamical theory of X-ray diffraction," Solid-State Phys., vol. 15, p. 53, 1963.

[15] B. W. Batterman and H. Cole, "Dynamical diffraction of X-rays by perfect crystals," Rev. Mod. Phys., vol. 36, pp. 681-717, 1964.

[16] J. W. M. DuMond, "Theory of the use of more than two successive X-ray crystal reflections to obtain increased resolving power," Phys. Rev., vol. 52, pp. 872-883, 1937.

[17] R. K. Smither and D. E. Roa, Crystal Diffraction Lens for Medical Imaging. San Diego, CA: SPIE-Int. Soc. Opt. Eng., 2000, vol. 3977, pp. 342-352.

[18] T. Johansson, "Über ein neuartiges genau fokusseriendes röntgenspektrometer," Zeitschrift für Physik, vol. 82, pp. 507-528, 1933.

[19] H. H. Johann, "Die erzeugung lichtstarker röntgenspektren mit hilfe von konkavkristallen," Zeitschrift für Physik, vol. 69, pp. 185-206, 1931.

[20] U. Bergmann and S. P. Cramer, High-Resolution Large-Acceptance Analyzer for X-Ray Fluorescence and Raman Spectroscopy. San Diego, CA: SPIE-Int. Soc. Opt. Eng., 1998, vol. 3448, pp. 198-209. 